\title{
A three DoFs wearable tactile display for exploration and manipulation of virtual objects
}

\author{
Francesco Chinello* \\ Department of Information Engineering, \\ University of Siena
}

\author{
Monica Malvezzi ${ }^{\dagger}$ \\ Department of Information Engineering, \\ University of Siena
}

\author{
Claudio Pacchierotti ${ }^{\ddagger}$ \\ Department of Information Engineering, \\ University of Siena
}

\author{
Domenico Prattichizzo $§$ \\ Department of Information Engineering, \\ University of Siena \\ Department of Advanced Robotics,
}

\begin{abstract}
This paper describes a wearable haptic display with small dimensions and low weight, that allows to simulate on the fingertip a wide range of contact forces. The device consists of two platforms: a static one, fixed on the back side of the finger, which supports three actuators and the mechanical instrumented system, and a mobile one, which interacts directly with the fingertip. The platforms are connected by three cables whose lengths and strains are regulated by the motors. Three force sensors, placed on the mobile platform, measure the actual forces applied to the finger. This work summarizes the design of the proposed display and presents a numerical model analysing the relationship between the forces registered at the fingertip and the platform's orientation and displacement. In order to validate the device an experiment of curvature discrimination has been carried out.
\end{abstract}

\section{INTRODUCTION}

This paper focuses on the problem of reproducing realistic contact force sensations due to the contact of the fingers with virtual objects and surfaces, by means of wearable haptic devices.

Several portable user interface devices with haptic feedback have been recently developed for entertainment purposes along with highly immersive virtual reality environments, such as CAVE [1], TWISTER [2] or the well-known game interface Wii Remote Control [3]. However, their haptic feedback capabilities are limited to vibrations only.

Glove-type haptic displays, such as Rutgers Master II [4] or the CyberGrasp [5], can provide force sensations to all five fingers of the hand simultaneously. However, these displays are complex, expensive and they focus on providing kinesthetic feedback, leaving out the tactile component from the grasping interaction.

No suitable method for providing haptic feedback through a small and portable/wearable display has been established so far [6].

K. Minamizawa et al., in [7], developed a wearable haptic display, able to simulate weight sensations of virtual objects. The approach is based on the novel insight that the deformation of the fingertip makes a reliable weight illusion even when the proprioceptive sensation is absent. Their proposed display was a two degrees of freedom (DoFs) system able to reproduce normal and tangential components of the contact force at the fingertip.

A similar device has been developed by N.S. Tsagarakis et al. in [8], where two motors were employed to feed back tangential forces to the user, in order to simulate relative lateral motion, in terms of direction and velocity, on the user's fingertips.

Although this kind of displays are portable and use a reduced number of actuators, they have not a closed loop force control and

\footnotetext{
*e-mail: chinello@dii.unisi.it

†e-mail: malvezzi@dii.unisi.it

¥e-mail: pacchierotti@dii.unisi.it

§e-mail: prattichizzo@dii.unisi.it
}

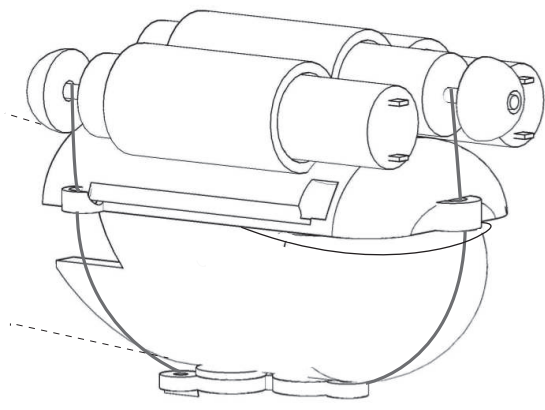

Figure 1: A sketch of the three DoFs wearable haptic display. The upper part is fixed on the back of the finger, while the lower platform is actuated by three wires connected to three small size electrical motors. In the lower platform three force sensors measure the force applied by the device to the fingertip.

cannot render forces in any directions. For example, the display described in [7] can reproduce the normal and lateral tangential component of the contact forces, while it is not able to apply forces in the longitudinal (pointing) tangential direction of the fingertip. As a consequence of such design, these devices are suitable for few grasping tasks.

More recently, M. Solazzi et al., in [9], developed a three DoFs wearable tactile display, but the portability and wearability of the device are limited by its mechanical structure. The motors are placed on the forearm and two cables for each actuated finger are necessary to transmit the motor torque to each fingertip.

This paper presents a wearable three DoFs haptic device (Fig. 1). It is able to perform stimuli on the fingertip by applying forces to the vertices of a rigid platform by means of cables whose lengths and strengths are regulated by three miniaturized DC motors. The contact force between the active part of the device and the fingertip is measured by three force sensors.

As the display developed in [7], this system provides tactile cutaneous stimuli and most of the kinesthetic feedback is missing. However, since the platform dimensions are very small, it can be easily integrated with other systems that also provide kinesthetic stimuli to the user's hand and arm. Possible solutions to compensate this lack of information have been presented in [10].

With respect to the device described in [7], the proposed one presents one additional actuated DoF, that allows to apply to the fingertip shear stimuli in both the tangential directions, thus presenting a wider applicability. Furthermore, the force sensors on the interface between the skin and the active part of the device are used in a closed loop control of force and position, that allows to increase the precision of the applied forces and to perform tests in which the applied stimulus changes dynamically.

The paper is organized as follows: the device is presented in Sec. 2 , Sec. 3 summarizes the kinematics and statics analysis of the de- 


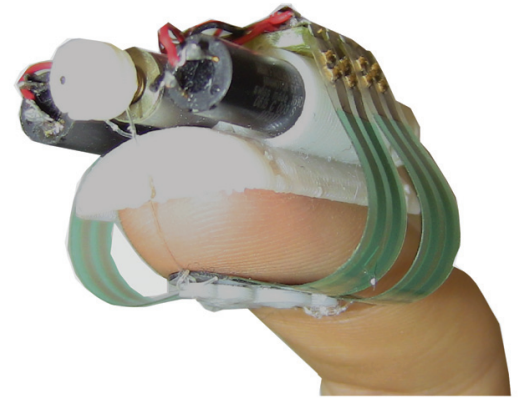

Figure 2: The three DoFs wearable haptic display prototype: the force sensors on the mobile platform measure the normal component of the force applied to the fingertip.

vice, represented as a three DoFs parallel mechanism [11], along with some solutions for the closed-loop control scheme. Some preliminary experiments carried out to validate the device and to verify its effectiveness in the reproduction of tactile sensations on the fingertip, are presented and discussed in Sec. 4. Finally Sec. 5 addresses concluding remarks and perspectives of the work.

\section{DEVICE DESCRIPTION}

Fig. 1 sketches the main idea of the proposed three DoFs wearable haptic device while a prototype of the device is shown in Fig. 2. It is composed of two main parts: one part is fixed to the back side of the finger and supports three small size DC electrical motors, while the active part is composed of a mobile contact patch placed on the fingertip's volar surface. The two main parts are connected by three wires whose lengths and strains are controlled by three DC motors. The actuators used for the device prototype are three 0615S Falhauber motors, with planetary gear-heads having 16:1 reduction ratio. Their main features are summarized in Table 1 .

The mobile platform has a Y shape and it allows to simulate contacts with generally oriented surfaces. The contact surface orientation can be modified by acting on the forces applied to the platform vertices.

Three piezoresistive force sensors $\left(400 \mathrm{FSR}^{\mathrm{TM}}\right.$ Interlink Electronics) are placed at the vertices, in contact with the finger, in order to measure the actual normal component of the force applied to the fingertips. The sensors have a diameter of $5 \mathrm{~mm}$ and a thickness of $0.3 \mathrm{~mm}$, making them very transparent to the user and easily embeddable in the device.

The mechanical supports for the actuators and the mobile platform are made using a special type of acrylonitrile butadiene styrene, called ABSPlus ${ }^{\mathrm{TM}}$ (Stratasys Inc.). The total weight of the whole device, including sensors, actuators, wires, and mechanical supports is about $30 \mathrm{~g}$.

The control of the device is managed by a microcontroller Atmega 328 installed on an Arduino Nano board which can control up to 6 PWM signals and read the outputs of up to 6 analog sensors.

\section{DeVice Model}

\subsection{The three DoFs platform}

In this section the kinematics and the statics of the device are presented. The mechanism can be modeled as a three DoFs parallel mechanism, where the static part is fixed on the back side of the finger, while the mobile platform, or end effector, is in contact with the finger pulp [11]. The mobile platform is moved acting on three cables connecting its vertices to three actuators. The kinematic scheme of the device is shown in Fig. 3. $B_{1}, B_{2}$, and $B_{3}$ are the points, on the platform, where the cables, linking the mobile

\begin{tabular}{|r|l|}
\hline Motor & 0615S \\
\hline Power & $0.12 \mathrm{~W}$ \\
\hline Stall torque (motor shaft) & $0.24 \mathrm{mNm}$ \\
\hline Friction torque & $0.02 \mathrm{mNm}$ \\
\hline Efficiency & $50 \%$ \\
\hline No load speed & $20000 \mathrm{rpm}$ \\
\hline Rotor inertia & $0.01 \mathrm{gcm}^{2}$ \\
\hline Maximum angular acceleration & $244 \cdot 10^{3} \mathrm{rad} / \mathrm{s}^{2}$ \\
\hline Diameter & $6 \mathrm{~mm}$ \\
\hline Length & $23 \mathrm{~mm}$ \\
\hline Weight & $2 \mathrm{~g}$ \\
\hline \hline Gearbox & $\mathbf{0 6} / \mathbf{1}$ \\
\hline \hline Reduction ratio & $16: 1$ \\
\hline Weight & $2.8 \mathrm{~g}$ \\
\hline Maximum output torque & $25 \mathrm{mNm}$ \\
\hline &
\end{tabular}

Table 1: Motor and gearhead main features.

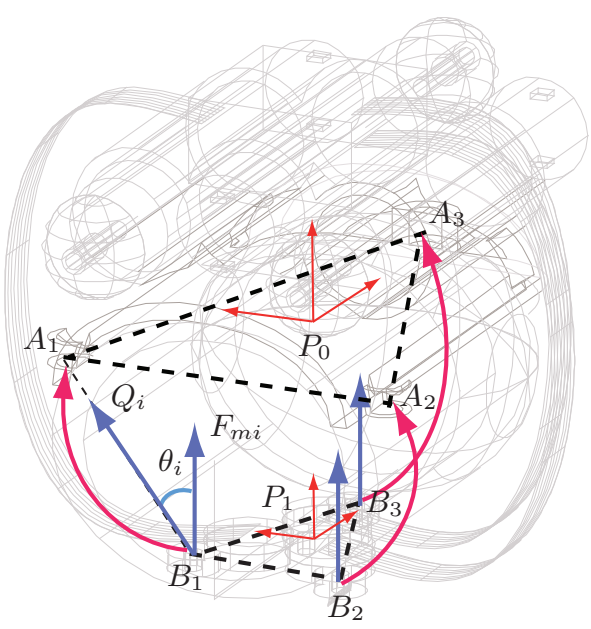

Figure 3: The three DoFs wearable haptic device kinematic scheme.

patch to the three actuators, pass and $S_{1}=\langle x, y, z\rangle$ is a reference frame fixed to the mobile platform whose origin $P_{1}$ is placed at the platform's geometric center, conventionally, chosen in the center of mass of the triangle defined by the points $B_{i}$.

Let $A_{1}, A_{2}$, and $A_{3}$ be the vertices of the fixed platform and $S_{0}=\langle X, Y, Z\rangle$ a reference frame on the fixed platform whose origin is located at $P_{0} . A_{i}$ and $B_{i}$ coordinates, expressed, respectively, in $S_{0}$ and $S_{1}$ reference frames are summarized in Tab. 2.

\subsection{Direct and inverse kinematics}

The transformation from frame $S_{1}$ to the fixed frame $S_{0}$ can be described by a vector $p=P_{1}-P_{0}$ and a $3 \times 3$ rotation matrix $R_{1}^{0}$, defined as a function of the yaw $(\gamma)$, pitch $(\beta)$ and roll $(\alpha)$ angles. Let $a_{i}, i=1,2,3$ represent the coordinates of point $A_{i}$, with respect to the frame $S_{0}$. Let $b_{i}^{0}, i=1,2,3$ be the coordinates of point $B_{i}$, with respect to the same frame, that can be expressed as $b_{i}=p+R_{1}^{0} b_{i}^{1}$, where $b_{i}^{1}$ represent the coordinates of the same points expressed with respect to frame $S_{1} . A_{i}$ and $B_{i}$ coordinates, expressed in the base and platform frame respectively, are summarized in Table 2 .

The inverse kinematics problem consists of finding the distance between the platforms' vertices $d_{i}=B_{i}-A_{i}$, for a given displacement $p$ and an angular configuration $\alpha, \beta$ and $\gamma$. The solution in 


\begin{tabular}{|c|r|r|r|}
\hline & $\mathrm{x}$ & $\mathrm{y}$ & $\mathrm{z}$ \\
\hline$A_{1}$ & -0.8 & -1.3 & 0 \\
\hline$A_{2}$ & 0.8 & -1.3 & 0 \\
\hline$A_{3}$ & 0 & 1.3 & 0 \\
\hline
\end{tabular}

A)

\begin{tabular}{|c|r|r|r|}
\hline & $\mathrm{x}$ & $\mathrm{y}$ & $\mathrm{z}$ \\
\hline$B_{1}$ & -0.9 & -0.6 & 0 \\
\hline$B_{2}$ & 0.9 & -0.6 & 0 \\
\hline$B_{3}$ & 0 & 0.6 & 0 \\
\hline
\end{tabular}

B)
Table 2: Coordinates of the platform points: a) fixed platform points $A_{i}$ with respect to $S_{0}[\mathrm{~cm}]$, b) mobile platform points $B_{i}$ with respect to $S_{1}[\mathrm{~cm}]$.

this case is straightforward

$$
q_{i}=\left\|d_{i}\right\|=\sqrt{a_{i}^{2}+b_{i}^{2}-2 a_{i} b_{i}} \quad i=1, \cdots, 3
$$

where $q_{i}=\left\|d_{i}\right\|$ is the distance between the $i$-th vertices. From the distance between the points on the fixed and mobile platform, and from the finger curvature radii $R_{i}$ (that can be approximately considered constant), we can evaluate the actual length of the cables $l_{i}$ as

$$
l_{i}=2 R_{i} \arcsin \left(\frac{q_{i}}{2 R_{i}}\right)
$$

\subsection{Differential kinematics}

For the velocity analysis, following the standard procedure described for instance in [11], we can express the joint variable time derivative $\dot{q}_{i}$ as

$$
\dot{q}_{i}=J_{p} v_{p}
$$

where $J_{p} \in \Re^{3 \times 6}$ is the Jacobian matrix, and $v_{p}=\left[\begin{array}{ll}\dot{p} & \omega_{b}\end{array}\right]^{\mathrm{T}} \in \Re^{6}$, in which $\omega_{p}$ represents the mobile platform angular velocity [11]. Furthermore, by differentiating eq. (2), it is possible to define a relationship between cable length time derivatives $i_{i}$ and $v_{p}$

$$
i_{i}=J_{l} v_{p}
$$

\subsection{Statics}

Let $w_{p}=\left[\begin{array}{ll}f_{p}^{\mathrm{T}} & m_{p}^{\mathrm{T}}\end{array}\right]^{\mathrm{T}} \in \Re^{6}$ be the wrench applied to the mobile platform (expressed with respect to $S_{0}$ ), and $Q=\left[\begin{array}{lll}Q_{1} & Q_{2} & Q_{3}\end{array}\right]^{\mathrm{T}}$ the vector of force (norms) applied to the cables by the actuators.

Using the Principle of Virtual Works to the mobile platform, neglecting the friction between the cable and the finger, we obtain:

$$
w_{p}=J_{l}^{\mathrm{T}} Q
$$

We observe that a generic wrench $w_{p}$ can be reproduced by the platform if it belongs to the $\Re^{6}$ subspace whose basis is defined by the columns of $J_{l}^{\mathrm{T}}$. In this case the corresponding cable forces can be calculated as

$$
Q=\left(J_{l}^{\mathrm{T}}\right)^{\#} w_{p}
$$

where $\left(J_{l}^{\mathrm{T}}\right)^{\#}$ is the pseudoinverse of the Jacobian transpose.

The mobile platform includes three force sensors as shown in Fig. 2. Since their sensing areas are placed next to the platform vertices, in this preliminary work we assumed that, approximately, the measured forces $F_{m i}, i=1,2,3$ are applied in $B_{1}, B_{2}$ and $B_{3}$ respectively. Some experimental tests showed that the sensor measures are quite well decoupled: actuating one motor once, we obtain a significant force measure on the corresponding sensor, while in the other two sensors the force variation is enough small to be neglected. We can then assume that the force sensors measure the component of each actuator force normal to the platform, and if we neglect the finger curvature radii we obtain the following approximated relationship

$$
F_{m i}=Q_{i} s_{i} k=Q_{i} \cos \theta_{i}
$$

where $k$ is the unit vector parallel to direction $z$, and $\theta_{i}$ is the angle between $z$ axis and $d_{i}$ vector (see Fig. 3 ). It is worth noting that the measure $F_{m i}$ depends both on the amplitude of the actuator force $Q_{i}$ and on the configuration of the mobile platform.

\subsection{Finger compliance}

The platform displacement $\xi=\left[\begin{array}{llllll}p_{x} & p_{y} & p_{z} & \alpha & \beta & \gamma\end{array}\right]^{\mathrm{T}}$ produces a deformation of the fingertip that leads to a contact stress distribution. In quasi static condition the stress distribution on the fingertip is balanced by the wrench applied by the platform $w_{p}$ [12].

In this section we discuss about the relationship between platform configuration $\xi$ and wrench $w_{p}$ applied by the platform to the fingertip.

The fingertip is composed of two skin layers (epidermis and dermis), subcutaneous tissue, arterial bone, and nail [13]. Different mathematical and numerical models of the fingertip have been proposed in the literature. In [14], for example, a 2D continuum fingertip model is described, in which the finger is approximated by an homogeneous, isotropic and incompressible elastic material. In [12] a model that incorporates both inhomogeneity and geometry of the fingertip is proposed. The undeformed fingertip is modeled as an axial symmetric ellipsoidal elastic membrane filled with a incompressible fluid with an internal pressure. Also in this case the model is $2 \mathrm{D}$ and an external load is applied to the finger through a flat surface. The model predicts a pulp force/displacement relationship that can be represented as a non linear hardening spring, i.e. whose stiffness increases with the applied load. Most of the displacement is reached when the load reaches $1 \mathrm{~N}$, which corresponds to a displacement of about $2 \mathrm{~mm}$. In [13] a 2D Finite Element model of the fingertip is presented: the skin was modeled as an hyperelastic and viscoelastic membrane, the subcutaneous layer was considered a biphase material.

The force/deformation behavior of the fingertips in the lateral, or shearing, direction, is studied in [15]. In this work the impedance characteristics of the fingertips in the tangential direction to the tip surface were experimentally measured. The simplified Kelvin model was adopted to describe the relationship between applied tangential force and finger deformation, and the impedance characteristics of the human fingertips were identified by means of experimental tests. The experiments showed that the fingertips have different stiffness properties in the shearing direction: the thumb is more stiff than the other fingers. Furthermore, the shearing stiffness depends on the force direction: the finger is more stiff in the pointing direction than in the lateral one. Actually the stress/strain behavior of the fingertip under shearing forces is non linear: in [16] the authors experimentally quantified the anisotropic and hysteretic behavior of the fingertip deformation under the application of tangential forces.

In this paper we consider a simplified model for the fingertip: a linear relationship between the resultant wrench and the platform displacement. In other terms we assume that the platform configuration $\xi$ is proportional to the wrench $w_{p}$

$$
\xi=K^{-1} w_{p}
$$

where $K \in \Re^{6 \times 6}$ is the fingertip stiffness matrix. In this preliminary study an isotropic elastic behaviour is assumed for all the components of the stiffness matrix: $K=k I, k=2 \mathrm{~N} / \mathrm{mm}$ [17]. A more detailed model that is currently being investigated uses different values for the stiffness components

$$
K=\operatorname{diag}\left[\begin{array}{llllll}
k_{t} & k_{t} & k_{n} & k_{b} & k_{b} & k_{s}
\end{array}\right]
$$


where $k_{t}$ is the skin stiffness in the tangential direction, or shear stiffness [15], $k_{n}$ is the finger stiffness in the normal direction [12], $k_{b}$ is the bending stiffness, that can be evaluated as a function of the normal stiffness $k_{n}$ and the contact patch dimension, and finally $k_{s}$ is the torsional stiffness (spin), that can be evaluated as a function of the shear stiffness $k_{t}$ and the contact patch dimensions.

\subsection{Wrench and platform configuration estimation}

From the above described kinematic and static analysis, a procedure for the on-line estimation of contact forces and platform configuration has been developed. Assume that the platform displacement during the experiment is small compared to the platform geometric dimensions, that the initial platform configuration $\xi(0)$ is known, and that the sampling time is small, so that the configuration variation between two consecutive integration steps is small, i.e., for a generic time step $j, \xi(j) \cong \xi(j-1)$.

The estimation algorithm is reported in the block diagram shown in Fig. 4 and summarized in the following steps: for each time sample $j$

- read from the sensors the normal component of the contact forces $F_{m, i}(j), i=1, \cdots, 3$;

- approximate the actuator forces $\hat{Q}_{i}$ as described in eq.(7);

- estimate platform wrench $\hat{w}_{p}(j)$ by means of eq. (5);

- estimate platform configuration $\hat{\xi}(j)$ by means of the compliant model defined in eq. (8);

- solve the inverse kinematic problem of the platform and find the angles $\hat{\theta}_{i}(j)$.

\subsection{Control}

From the control point of view, the device can be represented as a non linear, multi-input multi-output (MIMO) coupled system. In this preliminary work, two possible control strategies have been considered.

The first control scheme, shown in Fig. 5, is a force control where we want to regulate the cable forces $Q_{r}$ : the reference force $Q_{r}$ is compared to the estimated one $\hat{Q}$ and the error drives the PD controllers of the motors. The cable strengths $\hat{Q}$ are estimated from the force measures $F_{m, i}$, according to the procedure described in the preceding section and summarized in Fig. 4.

The second control scheme is a position control shown in Fig. 6, where the motors are regulated so that the mobile platform reaches a reference configuration $\xi_{r}$. The inverse kinematics of the parallel mechanism described in eq. (1) allows to evaluate the corresponding reference cable lengths $l_{r}$. The reference cable lengths are compared to the estimated one $\hat{l}$ and the error drives the the PD controllers of the motors. The cable lengths are not measured, but estimated: from the contact force normal components $F_{m, i}$,

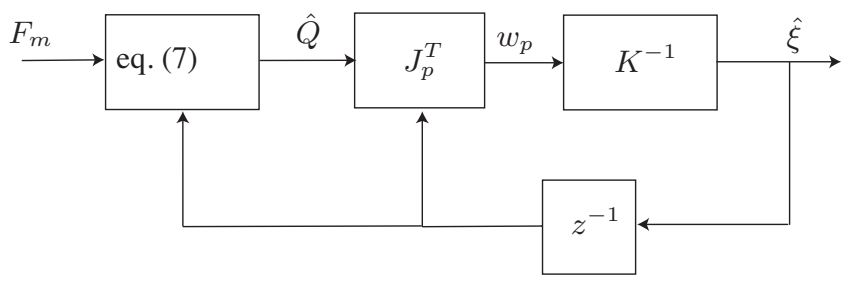

Figure 4: Block diagram of the procedure for the estimation of platform position $\hat{\xi}$ and wrench $\hat{w}_{p}$.

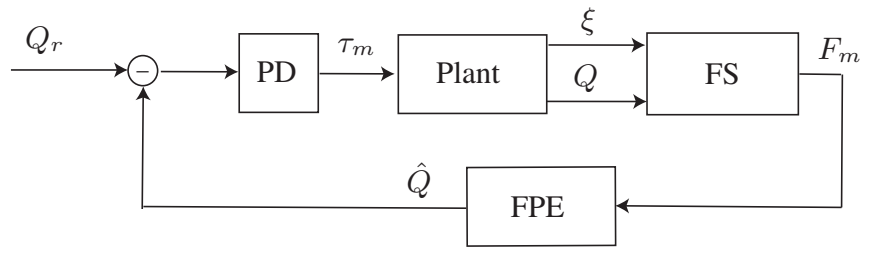

Figure 5: Block diagram of the device force control. The reference force $Q_{r}$ is compared to the estimated one $\hat{Q}$ : the error signal is the input for the DC motor PD controllers that generate the motor torque $\tau_{m}$. From the force $F_{m}$ measured by the sensors, indicated with the block FS (Force Sensors), and the platform position $\hat{\xi}$ estimated in the preceding time step, the Force and Position Estimation block, detailed in Fig. 4, evaluates the wire forces $\hat{Q}$.

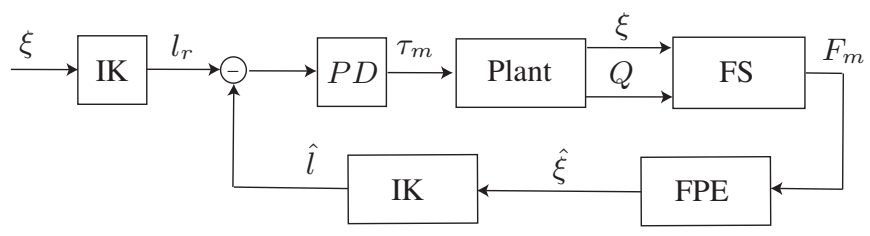

Figure 6: Block diagram of the platform position control system. From the reference position $\xi_{r}$, by means of the inverse kinematics procedure, represented by block IK, the reference wire lengths $l_{r}$ are estimated. Their values are compared to the estimated ones $\hat{q}$, evaluated applying the Inverse Kinematic procedure IK to the configuration $\hat{\xi}$ estimated in the FPE block, as a function of the measured forces $F_{m}$ and the position estimated in the preceding time step.

measured on the platform, the estimation procedure described in the preceding section and summarized in Fig. 4 allows to estimate the cable strains $\hat{Q}_{i}$ and the platform configuration $\hat{\xi}$. The inverse kinematics allows to estimate, from the configuration $\hat{\xi}$, the cable lengths $\hat{q}$.

In both control schemes, each motor is controlled by a closed loop chain with a PD controller. The reference signal is transmitted via a USB-to-serial converter interface with a sampling time of $0.01 \mathrm{~s}$ and stored in the Arduino register.

An application where the device with the force control scheme can be used is that described in [18] where a prototype of a joystick implementing a sensory substitution technique is proposed using tactile cutaneous device similar to those presented in this work but without force feedback control and consequently less accurate. Position control is suitable for applications in which the shape of the virtual contact surface is more important than the contact force. An example of this type of application is described in [19], in which the authors investigated the influence of tactile feedback in convex surface recognition tasks.

Both the control schemes are based on a force and position estimation procedure depending on the finger compliance model and referred as FPE in the block diagrams in Fig. 5 and 6. In this preliminary work we considered a linear model for the finger compliance, as those described in [15]. Work is in progress to investigate the sensitivity of the control performances on the finger compliance and to investigate the possibility of using different and more complex finger models.

To check the force control performance and accuracy two experimental tests were performed. Fig. 7 shows the control system performance when a step signal is applied to the reference values of the cable strengths $Q_{r}$. The reference force value was the same for each cable: $Q_{r, i}=0.3 \mathrm{~N}$, for $i=1, \cdots, 3$. In the figure, the 


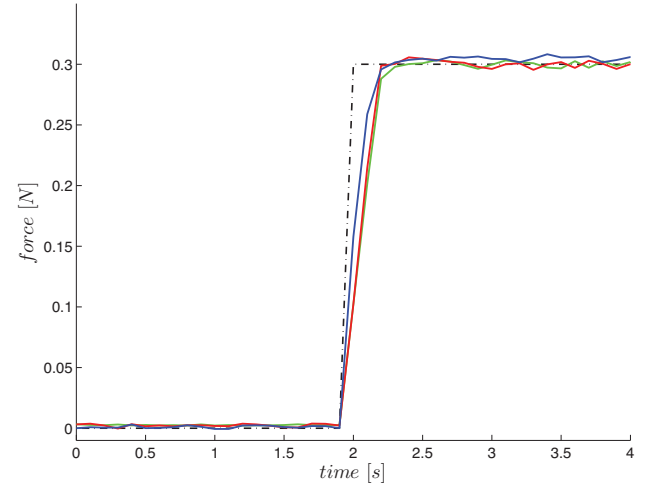

Figure 7: Force control step response of the three motors. The dashed black line represents the reference force value and the green, blue and red solid lines represent the estimated wire strains $\hat{Q}_{1}, \hat{Q}_{2}$ and $\hat{Q}_{3}$, respectively. The rising time is about 0.1 s and the steady state error is lower than $2 \%$.

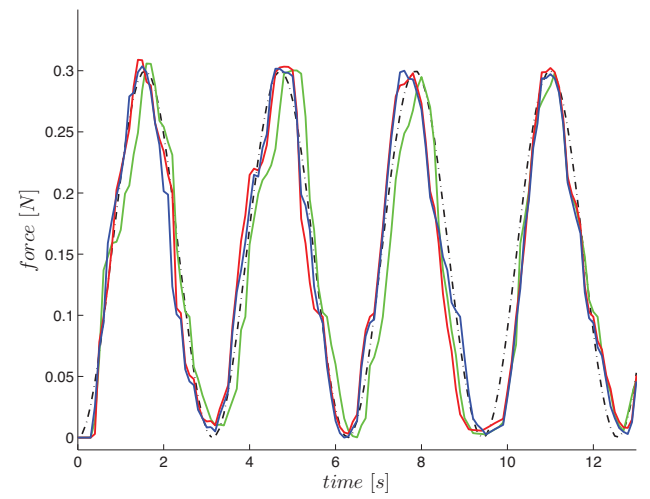

Figure 8: Response to a variable reference force signal. The reference signal is a sinusoidal function between $0 \mathrm{~N}$ and $0.3 \mathrm{~N}$. The dashed black line represents the signal reference and the green, blue and red lines represent estimated wire strains $\hat{Q}_{1}, \hat{Q}_{2}$ and $\hat{Q}_{3}$ respectively.

reference value (dashed) and the estimated cable strengths $\hat{Q}_{i}$ are shown. The test results show that the estimated forces reach the reference value with a rising time of about $0.1 \mathrm{~s}$ and an error in the stationary phase lower than $2 \%$. The system bandwidth is about $3.5 \mathrm{~Hz}$. Fig. 8 shows the behavior of the device when the force reference signal is sinusoidal: $Q_{r, i}=0.15 \sin (\pi t)+0.15 \mathrm{~N}$, for $i=1, \cdots, 3$.

\section{EXPERIMENT: CURVATURE DISCRIMINATION}

Inspired by the results on curvature discrimination in literature, where it has been proved that tactile feedback is important for surface recognition and that it is more relevant than kinesthesia for convex surface recognition $[19,20]$, we set up two experimental tests for surface discrimination using the proposed tactile device.

The operator wears one tactile device at the index finger. A virtual arc of a circle is then generated and virtually moved under the finger wearing the device. The operator is asked to keep the finger still and then tell how the curvature of the sphere changed from one trial to the other. The user is only able to see the position of the finger moving along a line, the same one in each trial, in order to know where the finger is with respect to the virtual sphere. The line does not provide any information about its curvature. Moreover she/he is

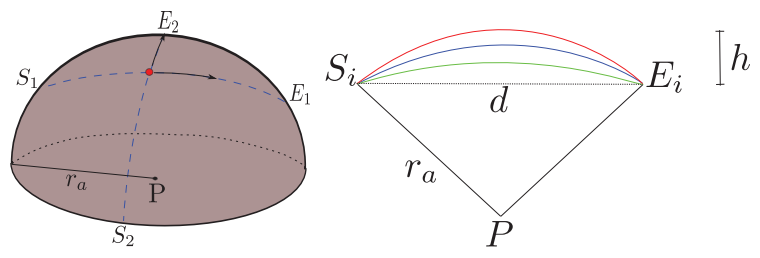

(a)

(b)

Figure 9: The convex curvature employed in the experiment. $d$ is fixed and $h$ changes thus changing the curvature of the virtual surface. In the experiments a virtual arc of a circle is generated under the finger wearing the device. In task A the surface is moving along $S_{1} E_{1}$ and in task B the surface is moved along $S_{2} E_{2}$.

not able to see her/his finger thus increasing the illusion of touching a virtual object ${ }^{1}$. The virtual sphere moves under the user's fingertip from $S_{i}$ to $E_{i}$ (see Fig. 9b). The device's actuators make the mobile platform tilt according to the curvature of the virtual surface thus giving the illusion of actually touching part of a sphere.

In the first experiment (task A) the virtual sphere moves from $S_{1}$ to $E_{1}$ while in the second experiment (task B) the sphere moves in the $S_{2}$ to $E_{2}$, as shown in Fig. 9a.

The virtual arc being moved under the fingertip is defined by the length of its chord $d$ and the height of the circular segment $h$ (Fig. $9 \mathrm{~b}$ ). Given $d$ and $h$, the radius of the circle $r_{a}$ and the length of the arc $l_{a}$ are

$$
\begin{aligned}
& r_{a}=\left(\frac{d^{2}}{4}+h^{2}\right) \frac{1}{2 h} \\
& l_{a}=\arcsin \left(\frac{d}{2 r_{a}}\right)
\end{aligned}
$$

Seven participants ( 6 males, 1 female, age range 20-25) took part to both the experiments, all of whom were right-handed. Five of them had previous experience with haptic interfaces. None of the participants reported any deficiencies in the perception abilities. Each participant made twelve repetitions of the curvature discrimination task, with four randomized trials for each curvature height $h_{i}$ :

$$
\begin{aligned}
& \text { - } h_{10}=10 \mathrm{~mm} \\
& \text { - } h_{20}=20 \mathrm{~mm} \\
& \text { - } h_{30}=30 \mathrm{~mm}
\end{aligned}
$$

The distance $d$ was fixed to $30 \mathrm{~cm}$ for all the trials. Fig. 10 shows the percentage of correct answers for each curvature and for each task. The collected data of each task passed the Shapiro-Wilk normality test. Then a parametric two-tailed paired t-test was performed, to evaluate the statistical significance of the differences between tasks (i.e. between the three curvatures). The $p$-values found reveal no statistically significant difference between the groups. It is worth reporting that no user confused the curvature with maximum height ( $\left.h_{30}\right)$ with the one with smallest height $\left(h_{10}\right)$ and vice versa.

This experiment confirms that is possible to discriminate different surface curvatures using the device here presented.

\section{Conclusion and Future Works}

In this work the design of a wearable tactile device for virtual exploration and manipulation is presented. In comparison to similar existing tactile devices, the one here presented has three actuated

\footnotetext{
${ }^{1} \mathrm{~A}$ short movie of the experiment can be downloaded at http://goo.gl/QYZcA
} 


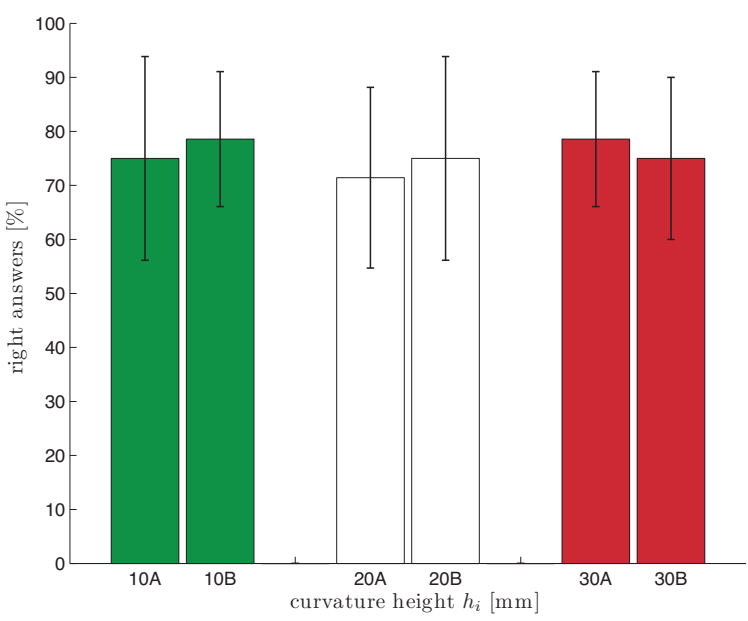

Figure 10: Percentage of correct answers for each curvature for each task. The results for both tasks for curvature $h_{10}, h_{20}, h_{30}$ are shown, respectively, in green, white, and red.

degrees of freedom and it is able to simulate a contact force with general direction at the fingertip.

The device can be represented as a three DoFs parallel mechanism in which a mobile platform is actuated modifying the strain of the three wires. Kinematics and statics of the device have been analyzed. The mobile platform is connected to the finger and applies a force whose direction and amplitude depends on cable strengths and on the platform position and orientation. The finger was modelled as a linear six dimensional spring. Future development of the presented study will include the analysis of other types of fingertip model.

Two control schemes were presented: in the first one (force control) the wire strains are controlled, in the second one (position control) the platform configuration (position and orientation) is controlled. Some preliminary tests on the force control performance showed that the dynamic response of the system is stable, and quite accurate. When a constant step reference signal is provided, the settling time is about $0.1 \mathrm{~s}$ and the steady state error is lower than $2 \%$. Furthermore these preliminary tests on the force control system showed a low sensitivity on the finger model.

In order to validate the device and verify its accuracy and effectiveness, some preliminary tests regarding the curvature discrimination of a virtual spherical surface have been performed.

We strongly believe that this highly-wearable device can be useful in many applications, ranging from rehabilitation to entertainment purposes and can bring haptic technologies to everyday life.

The device provides tactile cutaneous stimuli only, while most of the kinesthetic feedback is missing. Possible solutions to compensate for this lack of information, while preserving the portability of the device, are currently being investigated.

\section{ACKNOWLEDGEMENTS}

This work was partially supported by the Italian Ministry of Education, University and Research (MIUR) with PRIN 2008 Project "Underactuated systems for manipulazion in virtual environment" and by the European Commission with the Collaborative Project no. FP7-ICT-2009-6270460, "ACTIVE: Active Constraints Technologies for Ill-defined or Volatile Environments".

\section{REFERENCES}

[1] C. Cruz-Neira, D. Sandin, and T. DeFanti, "Surround-screen projection-based virtual reality: the design and implementation of the cave," in Proceedings of the 20th annual conference on Computer graphics and interactive techniques, pp. 135-142, ACM, 1993.

[2] S. Tachi, T. Maeda, Y. Yanagida, M. Koyanagi, and Y. Yokoyama, "A method of mutual tele-existence in a virtual environment," in Proceedings of ICAT 1996, pp. 9-18, 1996.

[3] Nintendo Co., Inc., "Wii Remote," 2006.

[4] G. C. Burdea, "Haptic feedback for virtual reality," Virtual Reality and Prototyping Workshop, vol. 2, pp. 17-29, June 1999.

[5] M. McLaughlin, G. Sukhatme, C. Shahabi, J. Hespanha, A. Ortega, and G. Medioni, "The haptic museum," in Proceedings of the EVA 2000 Conference on Electronic Imaging and the Visual Arts, 2000.

[6] M. Benali-Khoudja, M. Hafez, J. Alexandre, and A. Kheddar, "Tactile interfaces: a state-of-the-art survey," in Int. Symposium on Robotics, Citeseer, 2004.

[7] K. Minamizawa, S. Fukamachi, H. Kajimoto, N. Kawakami, and S. Tachi, "Gravity grabber: Wearable haptic display to present virtual mass sensation," ACM SIGGRAPH 2007 emerging technologies, 2007.

[8] N. Tsagarakis, T. Horne, and D. Caldwell, "Slip aestheasis: A portable 2d slip/skin stretch display for the fingertip," in Eurohaptics Conference, 2005 and Symposium on Haptic Interfaces for Virtual Environment and Teleoperator Systems, 2005. World Haptics 2005. First Joint, pp. 214-219, IEEE, 2005.

[9] M. Solazzi, A. Frisoli, and M. Bergamasco, "Design of a cutaneous fingertip display for improving haptic exploration of virtual objects," in RO-MAN, 2010 IEEE, pp. 1-6, IEEE.

[10] K. Minamizawa, D. Prattichizzo, and S. Tachi, "Simplified design of haptic display by extending one-point kinesthetic feedback to multipoint tactile feedback," in IEEE Haptic Symposium, (Waltham, Massachusetts, USA), 2010.

[11] L. W. Tsai, Robot Analysis, the mechanics of serial and parallel manipulators. John Wiley and Sons, Inc., 1999.

[12] E. Serina, E. Mockensturm, C. Mote Jr, and D. Rempel, "A structural model of the forced compression of the fingertip pulp," Journal of biomechanics, vol. 31, no. 7, pp. 639-646, 1998.

[13] J. Z. Wu, R. G. Dong, S. Rakheja, A. W. Schopper, and W. P. Smutz, "A structural fingertip model for simulating of the biomechanics of tactile sensation," Medical Engineering and Physics, vol. 26, no. 2, pp. $165-175,2004$.

[14] M. Srinivasan and K. Dankekar, "An investigation of the mechanics of tactile sense using two dimensional models of the primate fingertip," Transactions of the ASME, Journal of Biomechanical Engineering, vol. 118 , pp. $48-55,1996$.

[15] N. Nakazawa, R. Ikeura, and H. Inooka, "Characteristics of human fingertips in the shearing direction," Biological Cybernetics, vol. 82, pp. 207-214, 2000.

[16] Q. Wang and V. Hayward, "In vivo biomechanics of the fingerpad skin under local tangential traction," Journal of Biomechanics, vol. 40, pp. 851-860, 2007.

[17] K. Park, B. Kim, and S. Hirai, "Development of a soft-fingertip and its modeling based on force distribution," in Robotics and Automation, 2003. Proceedings. ICRA'03. IEEE International Conference on, vol. 3, pp. 3169-3174, IEEE, 2003.

[18] D. Prattichizzo, C. Pacchierotti, S. Cenci, K. Minamizawa, and G. Rosati, "Using a fingertip tactile device to substitute kinesthetic feedback in haptic interaction," in Haptics: Generating and Perceiving Tangible Sensations. Eurohaptics 2010, Lecture Notes in Computer Science, (Amsterdam, The Netherlands), pp. 125-130, Springer Verlag, 2010.

[19] M. Wijntjes, A. Sato, V. Hayward, and A. Kappers, "Local surface orientation dominates haptic curvature discrimination," IEEE transactions on haptics, pp. 94-102, 2009.

[20] S. Lederman and M. Taylor, "Fingertip force, surface geometry, and the perception of roughness by active touch," Attention, Perception, \& Psychophysics, vol. 12, no. 5, pp. 401-408, 1972. 